\title{
Effect of Cyclooxygenase(COX)-1 and COX- 2 inhibition on furosemide-induced renal responses and isoform immunolocalization in the healthy cat kidney
}

\author{
L. Pelligand ${ }^{1,4^{*}}$, N. Suemanotham² ${ }^{2}$ J. N. King ${ }^{3}$, W. Seewald ${ }^{3}$, H. Syme ${ }^{4}$, K. Smith $^{5}$, P. Lees ${ }^{1}$ and J. Elliott ${ }^{1}$
}

\begin{abstract}
Background: The role of cyclooxygenase(COX)-1 and COX-2 in the saluretic and renin-angiotensin responses to loop diuretics in the cat is unknown. We propose in vivo characterisation of isoform roles in a furosemide model by administering non-steroidal anti-inflammatory drugs (NSAIDs) with differing selectivity profiles: robenacoxib (COX-2 selective) and ketoprofen (COX-1 selective).

Results: In this four period crossover study, we compared the effect of four treatments: placebo, robenacoxib once or twice daily and ketoprofen once daily concomitantly with furosemide in seven healthy cats. For each period, urine and blood samples were collected at baseline and within $48 \mathrm{~h}$ of treatment starting. Plasma renin activity (PRA), plasma and urinary aldosterone concentrations, glomerular filtration rate (GFR) and $24 \mathrm{~h}$ urinary volumes, electrolytes and eicosanoids ( $\mathrm{PGE}_{2}, 6$-keto-PGF1 ${ }_{a}, \mathrm{Tx}_{2}$ ), renal injury biomarker excretions [N-acetyl-beta-D-glucosaminidase (NAG) and Gamma-Glutamyltransferase] were measured. Urine volume (24 h) and urinary sodium, chloride and calcium excretions increased from baseline with all treatments. Plasma creatinine increased with all treatments except placebo, whereas GFR was significantly decreased from baseline only with ketoprofen. PRA increased significantly with placebo and once daily robenacoxib and the increase was significantly higher with placebo compared to ketoprofen (10.5 \pm 4.4 vs $4.9 \pm 5.0 \mathrm{ng} \mathrm{m}^{-1} \mathrm{~h}^{-1}$ ). Urinary aldosterone excretion increased with all treatments but this increase was inhibited by $75 \%$ with ketoprofen and $65 \%$ with once daily robenacoxib compared to placebo. Urinary PGE $\mathrm{P}_{2}$ excretion decreased with all treatments and excretion was significantly lower with ketoprofen compared to placebo. Urinary $\mathrm{TxB}_{2}$ excretion was significantly increased from baseline only with placebo. NAG increased from baseline with all treatments. Immunohistochemistry on post-mortem renal specimens, obtained from a different group of cats that died naturally of non-renal causes, suggested constitutive COX-1 and COX-2 co-localization in many renal structures including the macula densa (MD).
\end{abstract}

Conclusions: These data suggest that both COX-1 and COX-2 could generate the signal from the MD to the renin secreting cells in cats exposed to furosemide. Co-localization of COX isoenzymes in MD cells supports the functional data reported here.

Keywords: Furosemide, Cyclooxygenase, COXib, NSAIDs, Robenacoxib, Ketoprofen, Feline, Renal, Macula densa, Renin-angiotensin-aldosterone system

\footnotetext{
*Correspondence: Ipelligand@rvc.ac.uk

'Department of Comparative Biomedical Sciences, The Royal Veterinary

College, Royal College Street, London NW1 OTU, UK

${ }^{4}$ Department of Clinical Sciences and Services, The Royal Veterinary College,

Hawkshead Lane, North Mymms, Hatfield AL9 7TA, Hertfordshire, UK

Full list of author information is available at the end of the article
} 


\section{Background}

The COXibs are a class of non-steroidal anti-inflammatory drugs (NSAIDs) which selectively inhibit cyclooxygenase (COX)-2, whilst sparing COX-1 activity. They were developed to provide analgesic and anti-inflammatory actions with greater safety than conventional NSAIDs, which are usually non-selective inhibitors [1]. Nevertheless, concerns over the renal safety COXibs have been raised [2]. Indeed, acute kidney injury, marked sodium retention and exacerbation of systemic hypertension have been reported with the use of COX-2 selective drugs in humans $[2,3]$.

$\mathrm{COX}$ isoforms exert key roles in the regulation of renal function through the production of protective prostaglandins (prostaglandin (PG) $\mathrm{E}_{2}$ and prostacyclin) [2, 4]. COX-1 is expressed in several renal cell types and is responsible for maintaining renal blood flow by attenuating vasoconstriction of the afferent arteriole (mediated by angiotensin II) through $\mathrm{PGE}_{2}$ or prostacyclin released locally [5]. Therefore, COX-1 inhibition by NSAIDs may precipitate ischaemic renal injury and decrease glomerular filtration rate (GFR) in hypovolaemic patients.

In several species COX-2 is constitutively expressed in a limited number of kidney cell types: the macula densa (MD) and cortical thick ascending loop and medullary interstitial cells $[6,7]$. The degree of renal COX-2 expression varies with age and disease state $[6,8,9]$, when renal homeostasis may become "prostaglandin dependent". Restricted COX-2 expression suggests differing functional roles in the cortex and medulla. Indeed, prostanoids derived from medullary COX are essential in promoting natriuresis [10], whereas cortical COX regulates renin release, which maintains GFR despite fluctuations in systemic arterial blood pressure [11]. Moreover, activation of the renin angiotensin aldosterone system (RAAS) stabilises arterial blood pressure and blood volume through vasoconstriction and increases sodium and water retention.

The reliance of renin release on COX-2 was demonstrated healthy human volunteers given furosemide (high renin state model) together with celecoxib (COX-2 selective) or indomethacin (non-selective) [12]. We do not know much about effects of COX inhibition in the feline kidney or the role of each isoenzyme in mediating natriuresis and RAAS activation or on the localisation of COX expression in cats with normal kidney function. Such information has clinical safety implications, as NSAIDs are used to treat pain in cats of all ages with normal or decreased plasma volume.

The aim of the present study was to elucidate localisation of COX-1 and COX-2 and identify their roles in stimulating renin secretion, secondary to a furosemide challenge in healthy cats. Of NSAIDs licensed for cats, two have differing COX-isoenzyme selectivities: robenacoxib (1 to $2 \mathrm{mg} \mathrm{kg}^{-1}$ oral, once daily) is a selective COX-2 inhibitor [13], whereas ketoprofen ( $1 \mathrm{mg} \mathrm{kg}^{-1}$ oral, once daily) is a selective COX-1 inhibitor [13, 14]. The study objectives were: (1) to determine the effects of COX-2 (robenacoxib) and COX-1 (ketoprofen) inhibition on furosemide-induced changes in the healthy cats, namely (i) diuretic and saluretic responses, (ii) GFR regulation, (iii) RAAS activation and (iv) possible nephrotoxicity; (2) to identify COX-1 and COX-2 immunolocalization in kidneys from cats with normal renal function.

\section{Results}

\section{In vivo furosemide study}

The mean doses of robenacoxib were $1.6 \mathrm{mg} \mathrm{kg}^{-1} \mathrm{~d}^{-1}$ for once daily dosing (range $=1.38-1.74$ ) and $3.2 \mathrm{mg} \mathrm{kg}$ ${ }^{-1} \mathrm{~d}^{-1}$ for dosing twice daily dosing( range $=2.76-$ $\left.3.36 \mathrm{mg} \mathrm{kg}^{-1} \mathrm{~d}^{-1}\right)$.

\section{Diuresis and natriuresis}

Twenty-four hour-corrected urine volumes were significantly increased from baseline with all treatments, by 139 , 94, 88 and $80 \%$ with placebo, once daily robenacoxib, twice daily robenacoxib and ketoprofen, respectively (Fig. 1a). For placebo, $24 \mathrm{~h}$-corrected urine output increased from $12.0 \pm 3.7$ to $28.8 \pm 12.8 \mathrm{~mL} \mathrm{~kg}^{-1} \mathrm{~d}^{-1}(P<0.001)$. Twentyfour hour urinary sodium excretion increased significantly in all treatment groups. For placebo, $24 \mathrm{~h}$ sodium excretion increased from $1.6 \pm 0.3$ to $2.7 \pm 1.3 \mathrm{mmol} \mathrm{kg}^{-1}$ day $^{-1}$ $(P=0.016$, Fig. 1b). Urinary chloride excretion increased with once daily robenacoxib $(P=0.025)$, twice daily robenacoxib $(P=0.03)$ and ketoprofen $(P=0.03)$ but not with placebo $(P=0.07)$ (Fig. 1c). Urinary $24 \mathrm{~h}$ potassium did not significantly increase with any treatment (Fig. 1d). Urinary calcium excretion significantly increased with all treatments.

\section{Haemoconcentration and GFR}

Plasma creatinine increased from baseline with once daily robenacoxib (143.1 vs $129.2 \mu \mathrm{mol} \mathrm{L} \mathrm{L}^{-1}, 11 \%$ increase, $P=0.029)$, twice daily robenacoxib (150.0 vs $127.6 \mu \mathrm{mol} \mathrm{L}{ }^{-1}, 17 \%$ increase, $\left.P<0.001\right)$ and ketoprofen (149.6 vs $133.3 \mu \mathrm{mol} \mathrm{L}{ }^{-1}, 12 \%$ increase, $P=0.017$ ) but not with placebo (Fig. 2a). For means of all treatments, there were numerically small but non-significant increases in packed cell volume ( $43.4 \pm 0.7 \%$ vs $39.7 \pm 1.0 \%$, Fig. $2 b)$ and plasma protein $\left(69.1 \pm 2.6\right.$ vs $66.7 \pm 1.5 \mathrm{~g} \mathrm{~L}^{-1}$, Fig. $\left.2 \mathrm{c}\right)$. A significant decrease in GFR from baseline was obtained only with ketoprofen $\left(1.45 \pm 0.25\right.$ vs $1.74 \pm 0.38 \mathrm{~mL} \mathrm{~kg}^{-1}$ $\min ^{-1}, P<0.01$, Fig. 1f).

\section{Effect on the renin angiotensin aldosterone system (RAAS)}

Plasma renin activity (PRA) increased significantly from baseline with placebo and once daily robenacoxib but not with twice daily robenacoxib or ketoprofen (Fig. 2d). The maximal placebo-associated PRA increase was reduced by 18,16 and $53 \%$ with the three 

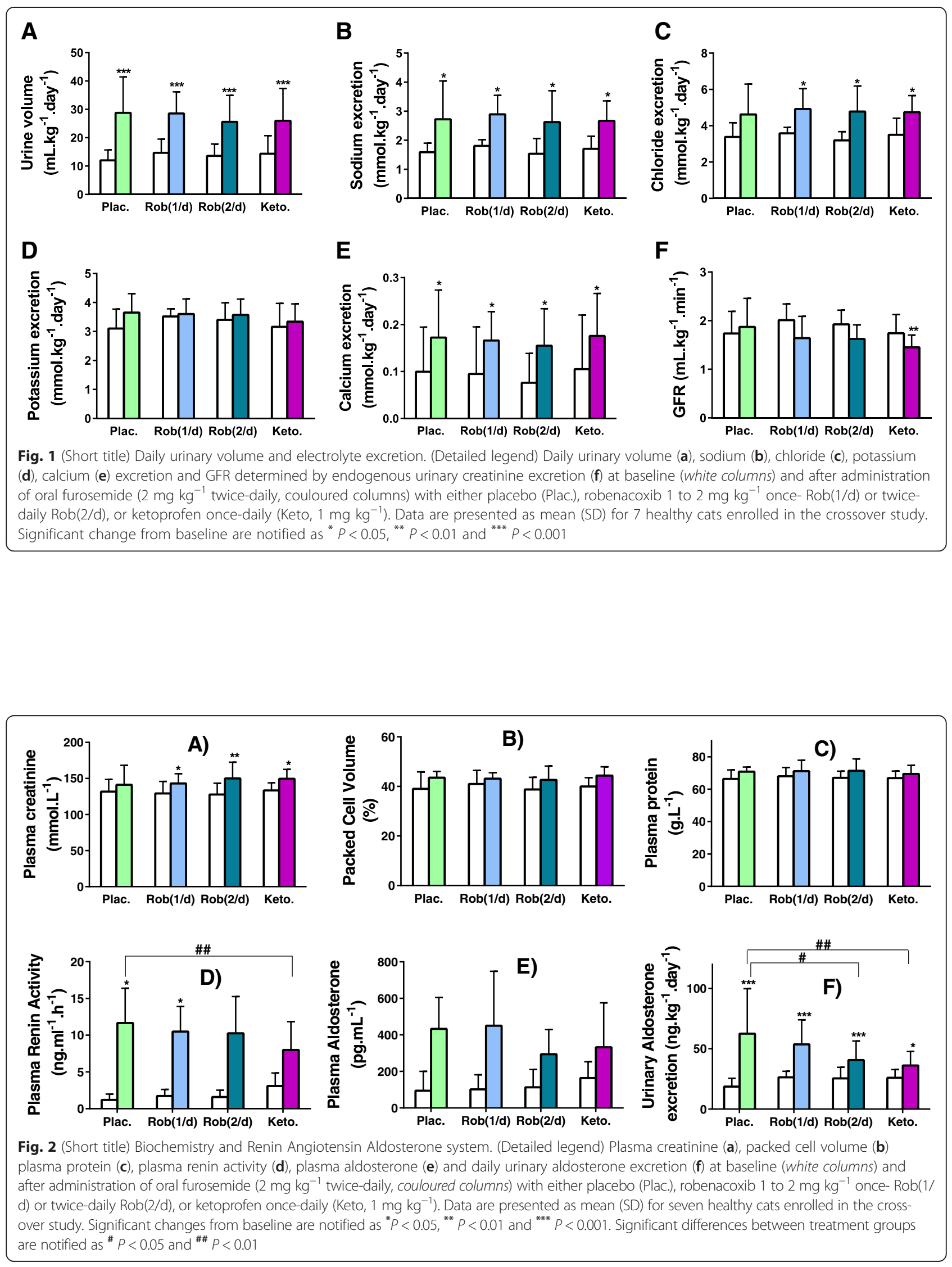
drug treatments, respectively. The increase in PRA from baseline was higher with placebo compared to ketoprofen $\left(10.5 \pm 4.4\right.$ vs $\left.4.9 \pm 5.0 \mathrm{ng} \mathrm{ml}^{-1} \mathrm{~h}^{-1}, P<0.01\right)$.

Plasma aldosterone concentration did not increase significantly from baseline with any treatment (Fig. 2e). However, inter-animal variability in differences between baseline and post-treatment concentrations was high. In contrast, urinary aldosterone excretion increased from baseline with all treatments $(P<0.001$ or $P<0.05$, Fig. 2f). The increase was greatest after placebo dosing and the magnitude of this maximal response was attenuated by $75 \%$ by ketoprofen $(P<0.01)$ and $65 \%$ by twice daily robenacoxib $(P<0.05)$.

\section{Urinary eicosanoid excretion}

The daily urinary excretion of $\mathrm{PGE}_{2}$ was significantly decreased with all treatments; the relative response was greater with ketoprofen $(70 \pm 6.1 \%)$ compared to placebo $\left(27 \pm 34.6 \%, P<0.05\right.$, Fig. 3a). $\mathrm{PGE}_{2}$ suppression was less and non-significant compared to placebo for robenacoxib groups (50 \pm 23.0 and $47 \pm 17.5 \%$ for once and twice daily dosing, respectively). The excretion of 6-keto-PGF1 $\alpha$ was unaffected with all treatments (Fig. 3c). Urinary $\mathrm{TxB}_{2}$ excretion increased significantly from baseline only with placebo $\left(6.4 \pm 2.8\right.$ vs $3.5 \pm 1.5 \mathrm{ng} \mathrm{kg}^{-1} \mathrm{~d}^{-1}, P<0.021$, Fig. $\left.3 \mathrm{~b}\right)$. This increase with placebo was attenuated by 27,41 and
$41 \%$ with once daily robenacoxib, twice daily robenacoxib and ketoprofen, respectively.

\section{Urinary protein and enzyme excretion}

Daily urinary protein concentration and $\gamma$-glutamyl transferase activity were not increased by any treatment (Fig. 3d and e). Mean urinary $\mathrm{N}$-acetyl-beta-D-glucosaminidase (NAG) activity for all treatments increased from baseline $(P=0.003$, Fig. $3 f)$ and there were no significant differences between treatments.

\section{Immunohistochemistry study}

\section{COX-1 expression in urinary tissues}

For control tissues, strong diffuse COX-1 positive immunostaining was seen in the cytoplasm and perinuclear area in the muscle of normal feline bladders. All negative control sections had no detectable immunostaining for COX-1 and COX-2.

The median [interquartile range] COX-1 expression scores of cats with normal kidneys are summarized in Table 1. The strongest immunopositive staining was observed in the thick and thin limbs of the loop of Henle and the collecting duct (Fig. 4a to d). Moderate binding of COX-1 occurred in the proximal tubule and MD. COX-1 staining was slight (weak positive in some cats and negative others) in the afferent arteriole and

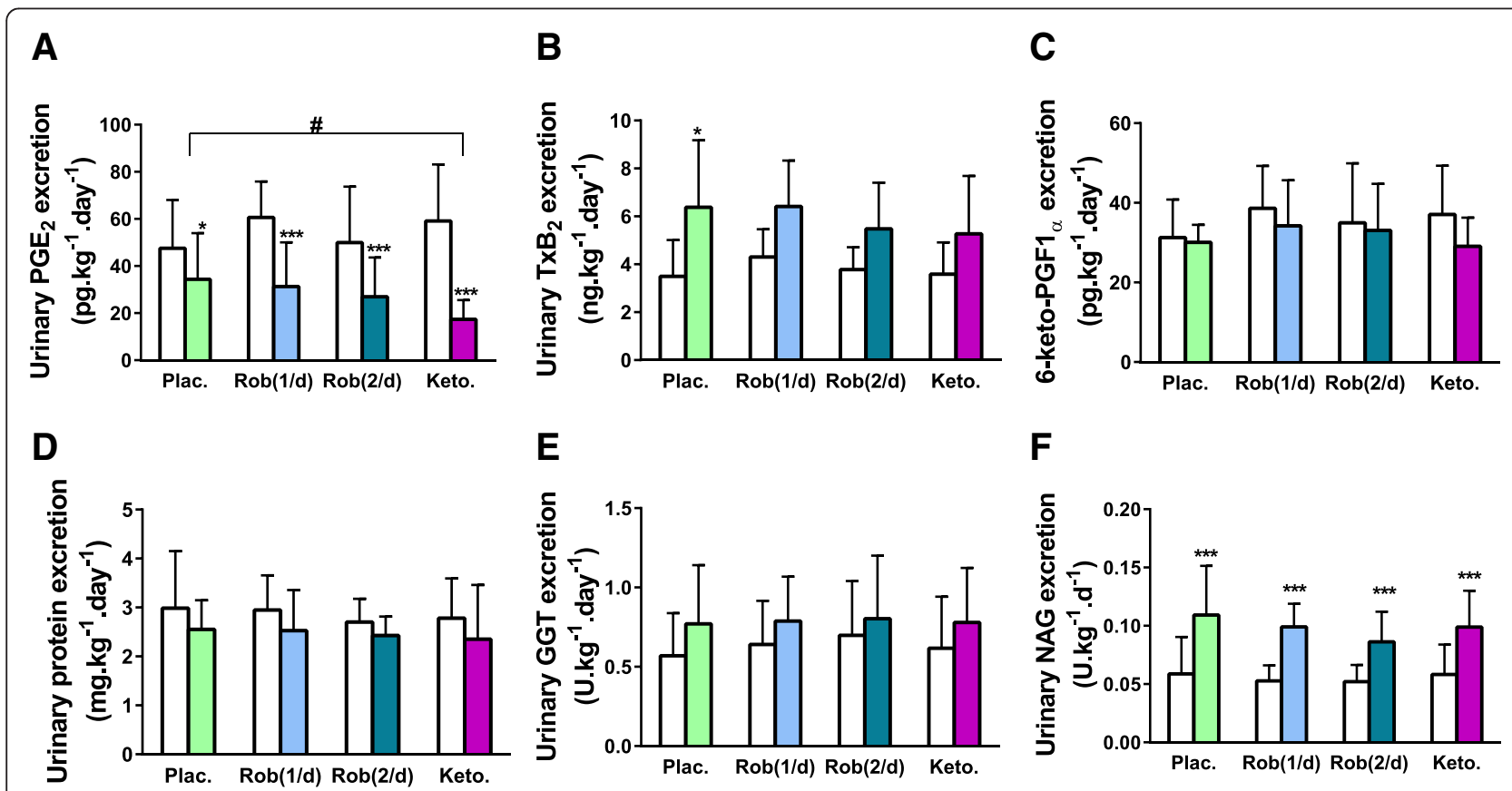

Fig. 3 (Short title) Daily urinary prostaglandin and enzyme excretion (activity). (Detailed legend) Daily urinary prostanoids excretion $\left[\mathrm{PGE}_{2}\right.$ (a), TxB 2 (b) and 6-ketoPGF $1_{a}(\mathbf{c})$ ], daily proteinuria $(\mathbf{d})$, gamma-Glutamyl transpeptidase (e) and N-acetyl-beta-D-glucosaminidase, NAG (f) urinary activities at baseline (white columns) and after administration of oral furosemide $\left(2 \mathrm{mg} \mathrm{kg}^{-1}\right.$ twice-daily, couloured columns) with either placebo (Plac.), robenacoxib 1 to $2 \mathrm{mg} \mathrm{kg}^{-1}$ once- Rob(1/d) or twice-daily Rob(2/d), or ketoprofen once-daily (Keto, $1 \mathrm{mg} \mathrm{kg}^{-1}$ ). Data are presented as mean (SD) for seven healthy cats enrolled in the crossover study. Significant change from baseline are notified as ${ }^{*} P<0.05$ and ${ }^{* * *} P<0.001$. Significant differences between treatment groups are notified as ${ }^{\#} P<0.05$ 
Table 1 COX-1 and COX-2 scores in the kidney of normal young and old cats

\begin{tabular}{lll}
\hline COX-1 immunostaining & Young $(n=10)$ & Old $(n=11)$ \\
\hline Thick limb & $1.37[1.01-1.66]$ & $1.75[1.43-1.81]$ \\
Collecting duct & $1.32[0.98-1.82]$ & $1.41[1.25-1.66]$ \\
Distal tubule & $1.10[0.84-1.35]$ & $1.14[1.00-1.43]$ \\
Thin limb & $0.97[0.58-1.20]$ & $1.42[0.82-1.55]$ \\
Macula densa & $0.75[0.55-0.81]$ & $0.82[0.80-1.13]$ \\
Proximal tubule & $0.56[0.44-0.71]$ & $0.74[0.36-0.91]$ \\
Glomerulus & $0.48[0.09-1.01]$ & $0.70[0.15-1.00]$ \\
Arteriole & $0.50[0.28-0.80]$ & $0.60[0.40-0.70]$ \\
Medullary interstitial cell & $0.00[0.0-0.25]$ & $0.00[0.00-0.00]$ \\
& & \\
COX-2 immunostaining & Young (n=10) & Old $(n=11)$ \\
Macula densa & $1.29[0.71-2.18]$ & $1.32[1.00-2.03]$ \\
Thin limb & $1.16[0.65-1.64]$ & $1.10[0.75-1.64]$ \\
Thick limb & $1.10[0.77-1.19]$ & $0.93[0.75-1.16]$ \\
Proximal tubule & $0.73[0.41-0.85]$ & $1.19[0.72-1.30]$ \\
Medullary interstitial cell & $1.00[0.00-1.00]$ & $1.00[0.00-1.00]$ \\
Collecting duct & $0.81[0.38-1.12]$ & $0.90[0.60-1.01]$ \\
Distal tubule & $0.45[0.27-1.13]$ & $0.86[0.42-1.09]$ \\
Glomerulus & $0.75[0.31-1.41]$ & $0.80[0.65-2.00]$ \\
Arteriole & $0.15[0.00-2.23]$ & $0.10[0.00-0.30]$ \\
\hline For each cat an average $]$ & &
\end{tabular}

For each cat, an average intensity score for each structure was calculated as described in the methods section (range 0 to 3 ). This single value has been used to calculate the median [interquartile range] value presented in the table for each group of cats. There was no difference in expression between young and old cats for either isoenzyme $(P>0.05)$

glomeruli in normal kidneys. COX-1 expression was undetectable in medullary interstitial cells. There was no statistically significant difference in COX-1 intensity scores between young and old cats.

\section{COX-2 expression in renal tissue}

Intense COX-2 positive immunostaining was seen consistently in the juxtaglomerular apparatus (JGA) of positive control sections. The regional immunohistochemical scores for COX-2 are summarised in Table 1. Positive COX-2 staining was highest in the MD, the thin and thick limb of the loop of Henle and the proximal tubule (Fig. 4e to h). COX-2 expression was usually depression was usually detectable in the glomeruli, the distal tubule and the collecting duct but only some of the arterioles. There were no statistically significant differences in COX-2 intensity scores between young and old cats.

\section{Discussion}

This is the first study to report the acute renal effects of COX-1 and COX-2 selective NSAIDs, administered at clinically recommended doses in combination with furosemide to healthy conscious cats.

\section{Validation of the furosemide model}

Furosemide (2 $\mathrm{mg} \mathrm{kg}^{-1}$ twice daily; clinically relevant dose) produced reliable diuretic and saluretic responses and stimulated the RAAS (increased PRA and plasma aldosterone concentrations).

Non-selective and COX-2 selective NSAIDs cause sodium retention in healthy humans [10]. Moreover, nonselective NSAIDs attenuate in vitro the furosemide-induced reduction in loop chloride reabsorption [15]. They also compete with furosemide for the organic acid transporter in proximal tubular cells, limiting its access to $\mathrm{NaK} 2 \mathrm{Cl}$ transporters [16]. In the present study, there was no inhibitory effect of acute NSAID administration on the diuretic and saluretic actions of furosemide; therefore electrolyte homeostasis in the cat may be regulated by mechanisms over-riding the acute COX blockade.

\section{Link between furosemide administration and increase in PRA}

There are two possible mechanisms to explain increased renin activity in response to furosemide in the present study [17]. First, by blocking $\mathrm{NaK} 2 \mathrm{Cl}$ transporter, furosemide removes the link between chloride concentration in the MD and renin secretion. Consequently, the MD signal for renin secretion persists regardless of the amount $\mathrm{NaCl}$ passing through the distal tubule [18], accounting for the increase in PRA. In these circumstances, basolateral cells of the MD of rabbit perfused kidneys increased COX-2 and $\mathrm{PGE}_{2}$ synthase expression $[19,20]$. The resulting $\mathrm{PGE}_{2}$ release constitutes the physiological mechanism of signal transmission from MD cells to the JGA [19]. This response regulates renin release and renal vascular resistance. Using a similar experimental setting to the present study, furosemide-induced renin release was blocked by COX-2 inhibitors in humans [12]. Second, the later occurring action of furosemide (due to the induced sodium and water depletion) is to reduce stimulation of the afferent arteriolar stretch receptors and carotid / aortic sinus baroreceptors, thus stimulating renin release $[12,17]$.

\section{Effects of NSAIDS on RAAS}

Ketoprofen significantly attenuated the furosemideinduced ten-fold increase in PRA in in this study, whereas the effect of robenacoxib was equivocal and probably dose dependent. An unavoidable limitation of the study is that ketoprofen (at the clinical dose rate and route selected) inhibits not only COX -1 for $24 \mathrm{~h}$, but also COX-2 for 10 to $11 \mathrm{~h}$ after oral administration (see integration of pharmacokinetic and pharmacodynamic data in Additional file 1: S2). Hence, attenuation of the PRA response may be due to inhibition of COX-1 or requires inhibition of both 

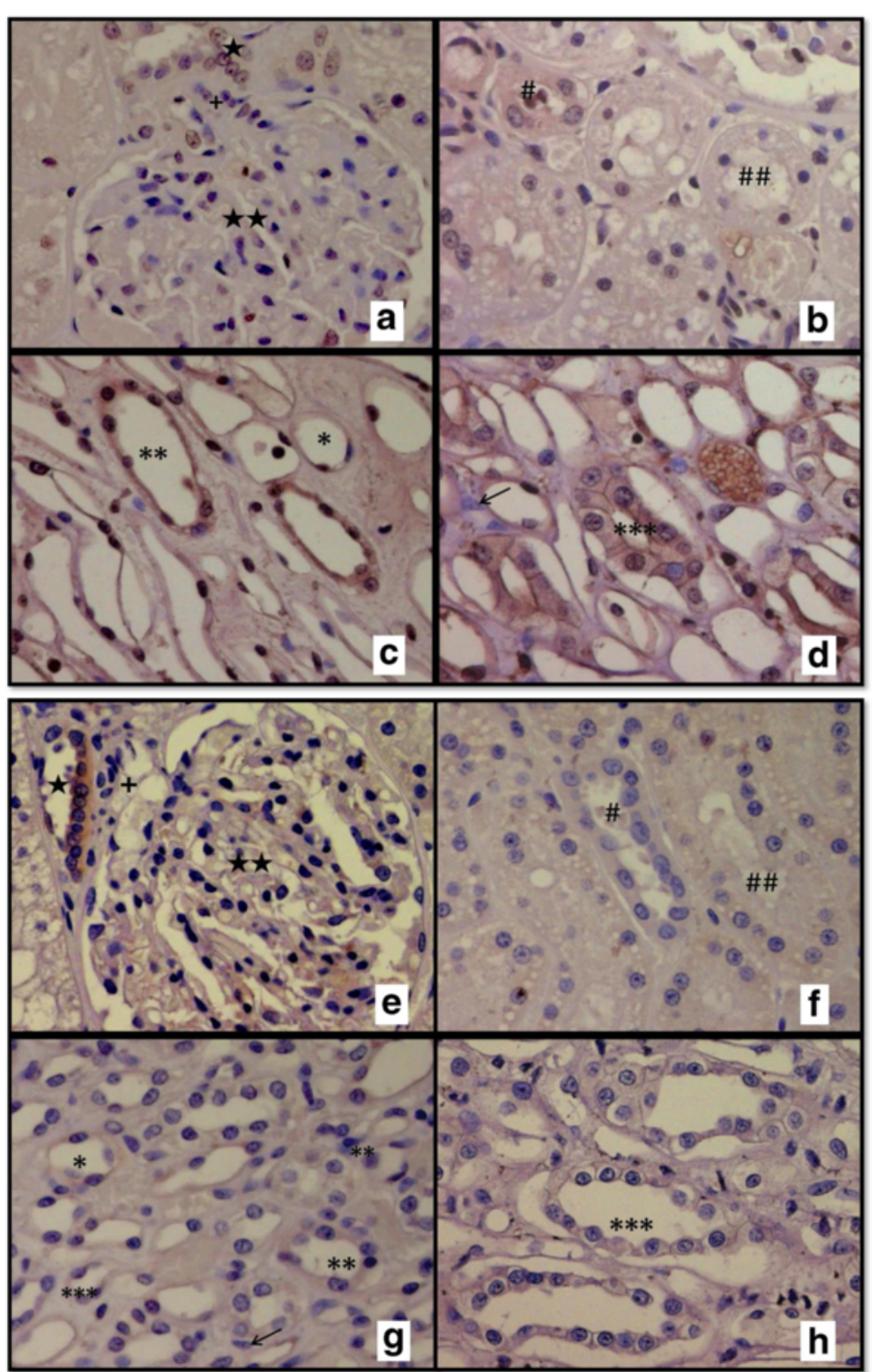

Fig. 4 (Short title) Renal localization of COX-1 and COX-2: (Detailed legend) COX-1 (Fig. 4.a to d, 4 top panels) and COX-2 (Fig. 4.e to h, 4 bottom panels) in the normal feline kidney. Results from 21 cats (ten young and 11 old). Magnification $\times 400$. denotes the macula densa, $\star \star$ denotes the glomerulus, + denotes the afferent arteriole, \# denotes the distal tubule, \#\# denotes the proximal tubule, ${ }^{*}$ denotes the thin limb of the loop of Henle, ${ }^{* *}$ denotes the thick limb of the loop of Henle, ${ }^{* *}$ denotes the collecting duct and denotes the medullary interstitial cells. a to d: The most intense expression of COX-1 was found in the thick limb of the loop of Henle (c) followed by the collecting duct (d), the distal tubule (b), the thin limb of the loop of Henle (c) and the macula densa (a), respectively. In the proximal tubule (b), the glomerulus (a) and the afferent arteriole (a), COX-1 expression was very weak to absent. h: The strongest COX-2 expression was found in the macula densa (e) followed by the thin loop of Henle (g), the thick limb of the loop of Henle $(\mathbf{g})$, the proximal tubule $(\mathbf{f})$, the medullary interstitial cells $(\mathbf{g})$ and the collecting duct $(\mathbf{h})$, respectively. In the distal tubule $(\mathbf{f})$, the glomerulus (e) and the afferent arteriole (e), COX-2 was either expressed very weakly or was absent

isoforms. Inhibition of COX-2 alone was insufficient to suppress this response. To elucidate the relative importance of COX-1 and COX-2 in mediating the PRA response to furosemide administration requires the use of a highly selective COX-1 inhibitor; no such compound is currently available.
Aldosterone release from the zona glomerulosa of the adrenal cortex is controlled by angiotensin II in circumstances of renal hypo-perfusion and increase in plasma potassium. Plasma potassium concentration did not change significantly in this study (results not shown), although the fractional excretion of potassium increased 
with all treatments, commensurate with the decrease in GFR. Plasma aldosterone concentration fluctuates over the course of the day, as aldosterone release can be affected by several factors, such as adrenocorticotropic hormone $(\mathrm{ACTH})$, body position, hyponatremia and atrial natriuretic peptide [21]. In the present study, the minor stress associated with restraint and blood sampling could have exerted an acute effect on ACTH and plasma aldosterone concentrations. The high inter-animal variability could account for the lack of significance of the numerical increase in plasma aldosterone after any of the treatment. Aldosterone excretion in urine collected over $24 \mathrm{~h}$ is, therefore, likely to provide a better indication of time-averaged aldosterone release than spot plasma aldosterone concentration measurement. Urinary aldosterone response was suppressed by both ketoprofen and twice daily robenacoxib and to a lesser extent by once daily robenacoxib. This supports a role for COX-2 but suggests that COX-1 is also involved in aldosterone secretion, as ketoprofen inhibited urinary aldosterone more than either dose of robenacoxib. Furthermore, the urinary aldosterone data are consistent with the PRA data, supporting the view that inhibition of both COX-1 and COX-2 is required to block the effect of furosemide in activating RAAS.

\section{Immunochemistry evidence for COX-1 and 2 in furosemide-induced PRA response}

Evidence for COX-2, and potentially also for COX-1, involvement in signalling from the MD cells to the renin secreting cells of the JGA, was obtained by immunohistochemistry, which demonstrated high level expression of COX-2 and weak expression of COX-1 within the $\mathrm{MD}$ of both young and older adult cats. In other species, the use of immunohistochemistry to localise COX-isoforms has demonstrated species and age-related differences in expression. Only COX-2 has been localised to the MD in most species, including dogs, rabbits and rats [7, 22, 23], but with both isoforms identified in mice [24]. Distribution of the isoenzymes in the other compartments of the feline kidney is similar to that seen in rodents, rabbits and dogs [7, 22, 23, 25]. In humans, COX-2 expression is reported in the MD in the foetus [26] and in subjects over 50 years of age [9], hence the inclusion of two age groups of cats in the present study. Kidney tissue was obtained from cats undergoing post-mortem examination for reasons other than CKD. These cats cannot, therefore, be described as healthy, and other features of their disease process might have altered renal tissue COX-enzyme expression. Nevertheless, the data indicate that COX-2 is the principal isoform present in cells of the feline $\mathrm{MD}$, whilst the finding of weak expression of COX-1 at this location suggests that interaction between the two isoforms may generate the signal to the JGA cells from the $\mathrm{MD}$ to secrete renin in some cats. This interpretation of the immunohistochemistry data is strengthened by the results of the functional studies which suggest involvement of both COX isoenzymes in signalling from MD to JGA to stimulate renin secretion.

\section{Vascular effects of furosemide and NSAIDs}

In the placebo group, the significant increase in plasma creatinine and slight but non-significant increases in packed cell volume and total protein suggest contraction of the volume of the central compartment by furosemide [27]. This may have led to reduced tonic stimulation of either the stretch receptors of the afferent arteriole or the cardiac or arterial baroreceptors, leading to release of renin, angiotensin II and aldosterone. However, systolic arterial blood pressure was not measured and therefore a baroreceptor mechanism cannot be confirmed. Altered stretch of afferent arteriole wall activates RAAS and the release of angiotensin II and aldosterone, leading to increased sodium reabsorption. Offset of the vasoconstrictive action of angiotensin II on the afferent arteriole and preservation of GFR is dependent on the local release of prostaglandins [4, 28]. GFR was significantly decreased with ketoprofen and approached significance for robenacoxib (both doses); it is therefore possible that the COX-1 dependent RAAS response to volume depletion was blunted with ketoprofen, accounting for the lesser effect of robenacoxib on furosemide-induced PRA increase.

\section{Effects of furosemide and NSAIDs on urinary eicosanoids}

Urinary eicosanoid excretion derives from local renal production. The effect of furosemide on urinary $\mathrm{PGE}_{2}$ excretion varies with species. It was unchanged in humans [12] but increased in dogs [29]. Surprisingly, in the present study furosemide reduced $\mathrm{PGE}_{2}$ excretion, probably through inhibition of $\mathrm{PGE}_{2}$ production. Against this background, it was not possible to relate $\mathrm{PGE}_{2}$ inhibition by NSAIDs to their action on furosemide-induced renin secretion. However, ketoprofen further reduced urinary $\mathrm{PGE}_{2}$ excretion, possibly indicating that renal COX-1 is the major source of urinary $\mathrm{PGE}_{2}$ in the cat. Attenuation of the furosemide increased urinary $\mathrm{TxB}_{2}$ excretion by ketoprofen and twice daily robenacoxib, compared to placebo, indicates that furosemide-induced renal $\mathrm{TxB}_{2}$ synthesis is probably the consequence of a COX-2 mediated compensatory vasoconstrictive mechanism.

\section{Effects of furosemide and NSAIDs on urinary protein excretion}

In dogs, increase in NAG index is associated with renal proximal tubular necrosis [30]. Furosemide significantly increased urinary NAG activity in all groups in the present study and the increases were not exacerbated by concomitant NSAID treatment. For all cats, NAG returned to baseline levels during the intervals between periods, 
indicating either a transient nephrotoxic effect of furosemide or, more likely, an increased lysosomal turnover in response to the increase in tubular fluid flow [31, 32].

\section{Methods}

\section{In vivo study design}

Seven neutered experimental cats (four males, three females, 2 years old), weighing $3.7 \pm 0.34 \mathrm{~kg}$, were enrolled in a four period crossover study. They were group housed except during sampling periods when they were individually housed in cages equipped with a timed urine collection system previously validated in cats [33]. Each cat was fed $25 \mathrm{~g}$ of a low sodium dry food twice daily (RF23, Royal Canin, Aimargues, France). Drinking water was available ad libitum. The study complied with the EU Directive 2010/63/EU (Project License Number 70/6132) and the protocol was approved by the Royal Veterinary College Ethics and Welfare Committee. All cats were declared healthy at the end of the study and subsequently re-homed.

At the beginning of each period, a pooled baseline urine sample was collected over $48 \mathrm{~h}$ during days 1,2 and 3 (Fig. 5). A baseline blood sample (cephalic or jugular venepuncture) was collected in the middle of the urinary collection interval (between 10.30 and 11.45 am on day 2). The treatments comprised administration of furosemide $2 \mathrm{mg} / \mathrm{kg}$ (Furosemide, Milpledge, Retfort, UK) orally twice daily $(08.00 \mathrm{am}, 07.00 \mathrm{pm})$ from the morning of day 6 to the morning of day 8 , concomitantly with one of four NSAID/placebo dosing regimens (Fig. 5): (1) robenacoxib (Onsior, $6 \mathrm{mg}$ flavoured tablets, Novartis Animal Health,
Camberley, UK,1 to $2 \mathrm{mg} \mathrm{kg}^{-1}$ ) once daily; (2) robenacoxib (1 to $2 \mathrm{mg} \mathrm{kg}^{-1}$ ) twice daily; (3) ketoprofen once daily (Ketofen, $5 \mathrm{mg}$ tablets, Merial, Harlow, UK, $1 \mathrm{mg} \mathrm{kg}^{-1}$ ); and (4) placebo once daily (active ingredient excluded). Robenacoxib tablets composition was $6.25 \%$ (6 mg) active ingredient, $30.0 \%$ microcryst. cellulose Avicel PH 101, $3.0 \%$ polyvinylpyrrolidone Kollidon K30, $43.75 \%$ yeast, 3.0 \% Crospovidone Kollidon CL, 12.0 \% yeast, $0.5 \%$ vanilla, $1.0 \%$ colloidal silicon dioxide (Aerosil 200), $0.5 \%$ magnesium stearate, makes a total weight of $96.0 \mathrm{mg}$ per tablet.

Furosemide, NSAIDs or placebo tablets were inserted in a small cube of moist cat food to facilitate oral administration. The rest of meal was withheld until at least 90 min after drug administration. Treatment order was randomised, following a Latin square design.

Further urine collection commenced after the first furosemide treatment and lasted for $48 \mathrm{~h}$ during days 6,7 and 8. Blood was collected on the morning of day 7 (from 10.30 to $11.45 \mathrm{am}, 2$ to $3 \mathrm{~h}$ after the morning treatment) in the same animal ranking order as day 2. A 10 day washout interval was allowed between the last administration of furosemide and collection of the baseline blood sample in the next period.

\section{Blood sampling, processing and assays}

Packed cell volume was measured before sample centrifugation $\left(10 \mathrm{~min}, 1500 \times \mathrm{g}, 20^{\circ} \mathrm{C}\right)$ and storage of plasma aliquots at $-80{ }^{\circ} \mathrm{C}$ until analysis. Plasma renin activity (Gamma Coat ${ }^{\circ}$, CA1553, DiaSorin Ltd, Bracknell, UK) was measured by radioimmunoassay, as validated by Syme et al. [34]. A radioimmunoassay validated in cats [30] was

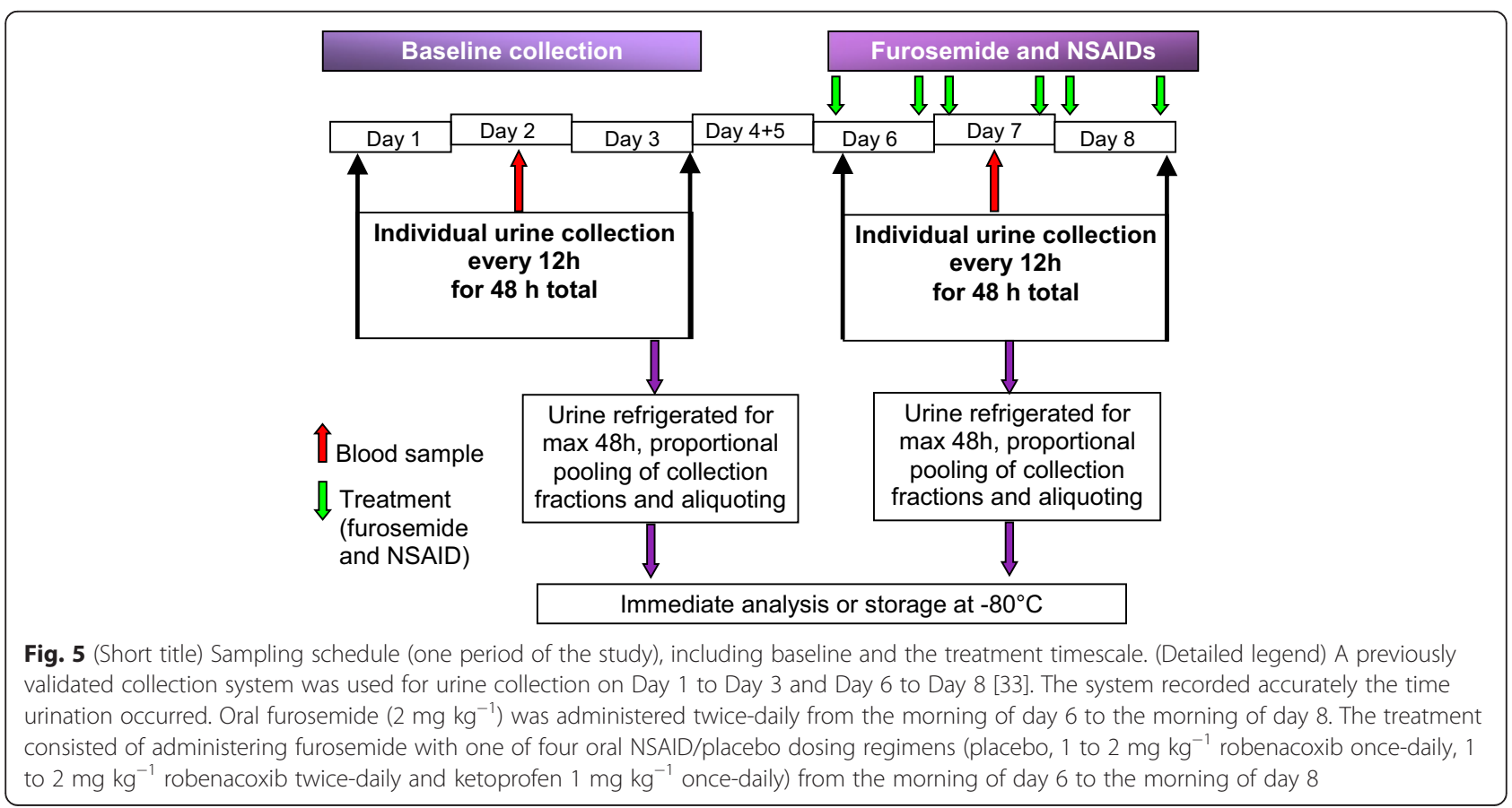


used to measured plasma aldosterone concentration (Coat-a-Count ${ }^{\ominus}$, Siemens Medical Solutions Diagnostics, Los Angeles, CA, USA). Creatinine (Jaffé colorimetric method), protein, sodium, potassium, chloride and calcium (ILAB 600, Idexx, Warrington, UK) concentrations were measured at a commercial laboratory.

\section{Urine sampling, processing and assays}

Urine was collected twice daily $(08.00 \mathrm{am}, 07.00 \mathrm{pm})$. Urinary specific gravity as recorded for each sample. Urine collected during the first day was stored at $4{ }^{\circ} \mathrm{C}$ for $24 \mathrm{~h}$ and pooled with urine from the second day. Accurate urination times and volumes were recorded to calculate $24 \mathrm{~h}$ averaged urinary volumes and analyte excretions over the collection period [33]. The $24 \mathrm{~h}$ urine excretion was corrected by the average percentage of voided volume lost through retention in the litter tray [33] and used alongside plasma and urinary creatinine concentrations to calculate GFR by endogenous urinary creatinine clearance. A nonselective COX inhibitor (ibuprofen $10 \mu \mathrm{M}$, Ref I7378, Sigma-Aldrich Co. Ltd, Poole, UK) solubilised in methanol was added to collected urine $(1: 1000 \mathrm{v} / \mathrm{v})$ to prevent $e x$ vivo eicosanoid production. Urine was centrifuged (1000 $g, 20{ }^{\circ} \mathrm{C}, 10 \mathrm{~min}$ ) and the supernatant stored at $-80{ }^{\circ} \mathrm{C}$. Urinary electrolytes (sodium, chloride, potassium, calcium) were measured using ion sensitive electrodes and creatinine and protein concentrations were measured using Jaffé and Lowry methods, respectively (ILab 600 analyser, Idexx). Urinary aldosterone concentration was measured by RIA after liquid extraction with dichloromethane. Urinary prostanoids were co-extracted on reverse phase extraction columns (Varian SepPak $200 \mathrm{mg}$ C18 LRC, Agilent) following a protocol adjusted from the manufacturer's recommendation. Urinary $\mathrm{Tx}_{2}$ concentration was measured by RIA $\left(\mathrm{TxB}_{2}\right.$ antibody, Ref P7291, Sigma and $\mathrm{TxB}_{2}$ Tracers, GE Healthcare biosciences Ltd, Little Chalfont, UK) and urinary $\mathrm{PGE}_{2}$ and 6-keto- $\mathrm{PGF}_{2 \alpha}$ were measured by enzymatic immunoassay (Immunoassays ADI-900-001 and ADI-900-004, Assay Designs, MI, USA). Urinary activity of $\gamma$-glutamyl transferase was measured on fresh samples within $24 \mathrm{~h}$ after the last collection using a colorimetric method ( $\gamma$-GT, Randox, Crumlin, UK). Urinary NAG was measured with a commercially available colorimetric assay ( $\mathrm{N}$-acetyl- $\beta$-glucosaminidase kit, no. 875406, Roche, Basel, Switzerland), validated in the cat [31]. Details of all assay protocols and validation results for blood and urine samples are presented in the Additional file 2: S1.

\section{Post-mortem study and specimen provenance}

Post-mortem kidney specimens were obtained (with informed owner consent) from 10 young ( $<5$ years) and 11 old ( $>9$ years) client-owned cats with normal renal function that had been euthanized or had died of natural non-renal causes. None of the cats had clinical signs indicative of chronic kidney disease (CKD), such as urinary specific gravity $<1.035$ or plasma creatinine $>177 \mu \mathrm{mol} \mathrm{L}^{-1}$.

\section{Immunohistochemistry protocols}

Three micrometre thick paraffin sections were dewaxed and rehydrated in grading ethanol followed by heating slides in a microwave at medium-high power in citrate buffer $\mathrm{pH} 6.0$ for $15 \mathrm{~min}$. Endogenous peroxidase activity was blocked by treating the tissue section with $3 \%$ hydrogen peroxide for $10 \mathrm{~min}$. Subsequently, $1.5 \%$ normal goat serum (Vector Laboratories, Burlingame, CA) or $1.5 \%$ normal donkey serum (Santa Cruz Biotechnology, Dallas, TX) was applied to block non-specific background staining of COX-1 and COX-2 primary antibodies, respectively. Slides were incubated with COX-1 (160108, Cayman Chemical, Camdridge, UK) or COX-2 (sc-1745, Santa Cruz Biotechnology) primary antibodies at 1:100 dilution at room temperature for $2 \mathrm{~h}$ and were then treated with biotinylated secondary antibody for COX-1 (BA-1000, Vector Laboratories) or COX-2 (sc-2023, Santa Cruz Biotechnology) for $1 \mathrm{~h}$. Immunoactive complexes were detected by incubating the sections with avidin-biotin complex system (Santa Cruz Biotechnology) for $30 \mathrm{~min}$ and visualized with diaminobenzidine. The sections were counterstained with hematoxylin. Negative control slides were prepared by replacing the primary antibody with $1.5 \%$ normal serum. Feline normal bladder sections were used as the COX-1 positive control [35], while canine foetal kidney sections were selected as the COX-2 positive control [36]. Stained sections were evaluated microscopically in a blinded manner and standard morphological criteria were used to identify the kidney structures. For each renal structure, staining intensity was assessed from 0 to 3 . In each cat, $10 \mathrm{MD}$, 10 tubules and 20 glomeruli were randomly assessed and the median [interquartile range] intensity score was calculated as previously described [37].

\section{Statistical analysis}

SAS software (Version 9.1.3, SAS Institute, Cary, NC, USA) was used for statistical analysis. When necessary, data were $\log$ transformed to satisfy the assumption of normal distribution of the residuals (Shapiro Wilk test) and homoscedasticity. An ANOVA model, containing sequence, period, sex, and baseline value as fixed effects and animal as a random effect, was fitted to all dependent variables. The effects of NSAID treatments were compared in a pairwise manner. The reported $p$-values are raw $p$ values corrected with a Tukey adjustment for multiple comparisons. In addition, the difference from baseline 
(or logarithmic equivalent) after furosemide for each NSAID treatment was tested against zero using a paired Student's $t$-test. Results are presented as mean \pm SD and $P<0.05$ was considered significant. For immunostaining studies, the Kruskal-Wallis test was used to compare the intensity scores between groups with significance level set at $P<0.05$.

\section{Conclusions}

The present study indicates the lack of effect of acute COX inhibition on furosemide-induced diuresis and natriuresis in cats. It is suggested both COX-1 and COX-2 are involved in the PRA and aldosterone response to furosemide and that COX-2 may be involved in regulating pathways other than angiotensin II stimulated aldosterone secretion. These findings, supported by the immunohistochemical localisation of COX-1 in the $\mathrm{MD}$ of the feline kidney, illustrate that renal physiology is species-dependent.

\section{Additional files}

Additional file 1 S1: PK-PD integration of robenacoxib and ketoprofen (description of data): estimation of the average duration of COX-1 and COX-2 inhibition achieved with robenacoxib once a day (RSID), robenacoxib twice a day (RBID) and ketoprofen (KET) using data from the literature. (DOCX $424 \mathrm{~KB}$ )

Additional file 2 S2: analytical method description and validation: (description of data) Validation data for Plasma Renin Activity, plasma and urinary aldosterone, urinary prostanoids and urinary enzymes. (DOCX $22 \mathrm{~kb})$

\section{Abbreviations}

COX: cyclooxygenase; GFR: glomerular filtration rate; NSAIDs: non-steroidal anti-inflammatory drugs; MD: macula densa; JGA: juxtaglomerular apparatus; PRA: plasma renin activity; $\mathrm{PGE}_{2}$ : prostaglandin E2; 6-keto-PGF1 ${ }_{\mathrm{a}}$ : 6-ketoprostaglandin $\mathrm{F}_{1}$ alpha (stable metabolite of Prostacyclin); $\mathrm{T}_{\mathrm{B}} \mathrm{B}_{2}$ : thromboxane $\mathrm{B}_{2}$; NAG: N-acetyl-beta-D-glucosaminidase.

\section{Competing interests}

L. Pelligand received a CASE award from BBSRC and Novartis Animal Health. J.N. King and W. Seewald are employees of Novartis Animal Health, now Elanco.

P. Lees, H. Syme and J. Elliott have acted as consultants to Novartis Animal Health.

\section{Authors' contributions \\ LP carried out the in vivo experiment, assays, and drafted the manuscript. NS carried out the immunohistochemistry studies and related statistics and contributed to manuscript drafting. JNK provided robenacoxib and placebo and supported the study. WS carried out the statistical analyses. HS provided pathology specimens and advised on data interpretation. KS provided control tissues for and assisted with the design of the immunohistochemistry protocol. PL designed the experiment and assisted with in vivo studies. JE designed the study and contributed to draft. All authors read and approved the final manuscript.}

\section{Acknowledgments}

In the memory of Hester Taylor for her skilled technical assistance with the cats. Novartis Animal Health funded this study (in vivo phase) and helped with the statistical analysis and writing up of the paper.

\section{Author details}

'Department of Comparative Biomedical Sciences, The Royal Veterinary College, Royal College Street, London NW1 OTU, UK. ${ }^{2}$ Faculty of Veterinary Science, Mahidol University, Nakhon Pathom, Thailand. ${ }^{3}$ Novartis Animal Health Inc., Clinical Development, Basel, Switzerland. ${ }^{4}$ Department of Clinical Sciences and Services, The Royal Veterinary College, Hawkshead Lane, North Mymms, Hatfield AL9 7TA, Hertfordshire, UK. ${ }^{5}$ Department of Pathology and Pathogen Biology, The Royal Veterinary College, Hawkshead Lane, North Mymms, Hatfield AL9 7TAHertfordshire, UK.

Received: 28 May 2015 Accepted: 18 November 2015

Published online: 03 December 2015

\section{References}

1. Warner TD, Giuliano F, Vojnovic I, Bukasa A, Mitchell JA, Vane JR. Nonsteroid drug selectivities for cyclo-oxygenase-1 rather than cyclo-oxygenase-2 are associated with human gastrointestinal toxicity: a full in vitro analysis. Proc Natl Acad Sci U S A. 1999;96(13):7563-8.

2. Giovanni G, Giovanni P. Do non-steroidal anti-inflammatory drugs and COX-2 selective inhibitors have different renal effects? J Nephrol. 2002;15(5):480-8.

3. Hippisley-Cox J, Coupland C. Risk of myocardial infarction in patients taking cyclo-oxygenase-2 inhibitors or conventional non-steroidal antiinflammatory drugs: population based nested case-control analysis. B M J. 2005;330(7504):1366.

4. Harris RC, Breyer MD. Physiological regulation of cyclooxygenase-2 in the kidney. Am J Physiol. 2001;281(1):F1-11.

5. Rose B, Post T. Effects of hormones on the renal function. In: Rose BD, Post TD, editors. Clinical Physiology of Acid-base and Electrolyte Disorders, vol. 2001. Fifthth ed. New York: McGraw-Hill Publishers; 2001. p. 163-238.

6. Khan KN, Stanfield KM, Dannenberg A, Seshan SV, Baergen RN, Baron DA, et al. Cyclooxygenase-2 expression in the developing human kidney. Pediatr Dev Pathol. 2001;4(5):461-6.

7. Khan KN, Venturini CM, Bunch RT, Brassard JA, Koki AT, Morris DL, et al. Interspecies differences in renal localization of cyclooxygenase isoforms: implications in nonsteroidal antiinflammatory drug-related nephrotoxicity. Toxicol Pathol. 1998;26(5):612-20.

8. Komhoff M, Grone HJ, Klein T, Seyberth HW, Nusing RM. Localization of cyclooxygenase-1 and -2 in adult and fetal human kidney: implication for renal function. Am J Physiol. 1997;272(4 Pt 2):F460-468.

9. Nantel F, Meadows E, Denis D, Connolly B, Metters KM, Giaid A. Immunolocalization of cyclooxygenase-2 in the macula densa of human elderly. FEBS Lett. 1999:457(3):475-7.

10. Catella-Lawson F, McAdam B, Morrison BW, Kapoor S, Kujubu D, Antes L, et al. Effects of specific inhibition of cyclooxygenase-2 on sodium balance, hemodynamics, and vasoactive eicosanoids. J Pharmacol Exp Ther. 1999; 289(2):735-41.

11. Hao CM, Breyer MD. Physiologic and pathophysiologic roles of lipid mediators in the kidney. Kidney Int. 2007;71(11):1105-15.

12. Stichtenoth DO, Marhauer V, Tsikas D, Gutzki FM, Frolich JC. Effects of specific COX-2-inhibition on renin release and renal and systemic prostanoid synthesis in healthy volunteers. Kidney Int. 2005;68(5):2197-207.

13. Schmid VB, Seewald W, Lees P, King JN. In vitro and ex vivo inhibition of COX isoforms by robenacoxib in the cat: a comparative study. J Vet Pharmacol Ther. 2010;33(5):444-52.

14. Pelligand L, King JN, Hormazabal V, Toutain PL, Elliott J, Lees P. Differential pharmacokinetics and pharmacokinetic/pharmacodynamic modelling of robenacoxib and ketoprofen in a feline model of inflammation. J Vet Pharmacol Ther. 2014;37(4):354-66.

15. Kirchner KA. Indomethacin antagonizes furosemide's intratubular effects during loop segment microperfusion. J Pharmacol Exp Ther. 1987;243(3):881-6.

16. Kocheval D, Scott M: Drugs affecting renal function and fluid-electrolyte balance. In Veterinary Pharmacology and Therapeutics, Ninth Edition, Eds Riviere, JE, Papich MG, Wiley Blackwell Publishers. 2009: 656-659.

17. Corsini WA, Hook JB, Bailie MD. Control of renin secretion in the dog. Effects of furosemide on the vascular and macula densa receptors. Circ Res. 1975; 37(4):464-70.

18. He XR, Greenberg SG, Briggs JP, Schnermann J. Effects of furosemide and verapamil on the $\mathrm{NaCl}$ dependency of macula densa-mediated renin secretion. Hypertension. 1995;26(1):137-42. 
19. Peti-Peterdi J, Komlosi P, Fuson AL, Guan Y, Schneider A, Qi Z, et al. Luminal $\mathrm{NaCl}$ delivery regulates basolateral PGE2 release from macula densa cells. J Clin Invest. 2003;112(1):76-82.

20. Mann B, Hartner A, Jensen BL, Kammerl M, Kramer BK, Kurtz A. Furosemide stimulates macula densa cyclooxygenase-2 expression in rats. Kidney Int. 2001:59(1):62-8

21. Rose B, Post T. Hypovolemic states. In: Rose BD, Post TD, editors. Clinical Physiology of Acid-base and Electrolyte Disorders, vol. 2001. Fifthth ed. New York: McGraw-Hill Publishers; 2001. p. 415-46.

22. Schumacher K, Castrop H, Strehl R, de Vries U, Minuth WW. Cyclooxygenases in the collecting duct of neonatal rabbit kidney. Cell Physiol Biochem. 2002;12(2-3):63-74.

23. Campean V, Theilig F, Paliege A, Breyer M, Bachmann S. Key enzymes for renal prostaglandin synthesis: site-specific expression in rodent kidney (rat, mouse). Am J Physiol. 2003;285(1):F19-32.

24. Meskell M, Ettarh R. Immunohistochemical localisation of renal cyclooxygenase-1 expression in non-steroidal anti-inflammatory drugtreated mice. Exp Toxicol Pathol. 2011;63(1-2):39-42.

25. Harris RC, McKanna JA, Akai Y, Jacobson HR, Dubois RN, Breyer MD. Cyclooxygenase-2 is associated with the macula densa of rat kidney and increases with salt restriction. J Clin Invest. 1994;94(6):2504-10.

26. Therland KL, Stubbe J, Thiesson HC, Ottosen PD, Walter S, Sorensen GL, et al. Cycloxygenase-2 is expressed in vasculature of normal and ischemic adult human kidney and is colocalized with vascular prostaglandin E2 EP4 receptors. J Am Soc Nephrol. 2004;15(5):1189-98.

27. Friedman PA, Roch-Ramel F. Hemodynamic and natriuretic effects of bumetanide and furosemide in the cat. J Pharmacol Exp Ther 1977:203(1):82-91.

28. Rose B, Post T. Regulation of the effective circulating volume. In: Rose BD, Post TD, editors. Clinical Physiology of Acid-base and Electrolyte Disorders. Fifthth ed. New York: McGraw-Hill Publishers; 2001. p. 258-84.

29. Sreenivasan V, Walker B, Krasney J, Mookerjee B, Venuto R. Role of endogenous prostaglandins in volume expansion and during furosemide infusion in conscious dogs. Hypertension. 1981;3(1):59-66.

30. Yu S, Morris JG. Plasma aldosterone concentration of cats. Vet J. 1998;155(1):63-8.

31. Jepson RE, Vallance C, Syme HM, Elliott J. Assessment of urinary N-acetylbeta-D-glucosaminidase activity in geriatric cats with variable plasma creatinine concentrations with and without azotemia. Am J Vet Res. 2010;71(2):241-7.

32. Jakobsen JA, Nossen JO, Jorgensen NP, Berg KJ. Renal tubular effects of diuretics and X-ray contrast media. A comparative study of equimolar doses in healthy volunteers. Invest Radiol. 1993;28(4):319-24.

33. Pelligand $L$, Lees $P$, Elliott J. Development and validation of a timed urinary collection system for use in the cat. Lab Anim. 2011:45(3):196-203.

34. Syme H. Studies of the Epidemiology and Aetiology of Systemic Hypertension in the Cat. London: London Royal Veterinary College; 2003.

35. Hayes A, Scase T, Miller J, Murphy S, Sparkes A, Adams V. COX-1 and COX-2 expression in feline oral squamous cell carcinoma. J Comp Pathol. 2006;135(2-3):93-9.

36. Heller DA, Fan TM, de Lorimier LP, Charney SC, Barger AM, Tannehill-Gregg $\mathrm{SH}$, et al. In vitro cyclooxygenase-2 protein expression and enzymatic activity in neoplastic cells. J Vet Intern Med. 2007;21(5):1048-55.

37. Sabbatini M, Vitaioli $L$, Baldoni $E_{1}$ Amenta F. Nephroprotective effect of treatment with calcium channel blockers in spontaneously hypertensive rats. J Pharmacol Exp Ther. 2000:294(3):948-54.

\section{Submit your next manuscript to BioMed Central and we will help you at every step:}

- We accept pre-submission inquiries

- Our selector tool helps you to find the most relevant journal

- We provide round the clock customer support

- Convenient online submission

- Thorough peer review

- Inclusion in PubMed and all major indexing services

- Maximum visibility for your research

Submit your manuscript at www biomedcentral com/submit
C BioMed Central 